\title{
10.
}

\section{ON THE THEORY OF ALGEBRAIC CURVES.}

[From the Cambridge Mathematical Journal, vol. IV. (1843), pp. 102-112.]

Suppose a curve defined by the equation $U=0, U$ being a rational and integral function of the $m^{\text {th }}$ order of the coordinates $x, y$. It may always be assumed, without loss of generality, that the terms involving $x^{m} ; y^{m}$, both of them appear in $U$; and also that the coefficient of $y^{m}$ is equal to unity: for in any particular curve where this was not the case, by transforming the axes, and dividing the new equation by the coefficient of $y^{m}$, the conditions in question would become satisfied. Let $H_{m}$ denote the terms of $U$, which are of the order $m$, and let $y-\alpha x, y-\beta x \ldots y-\lambda x$ be the factors of $H_{m}$. If the quantities $\alpha, \beta \ldots \lambda$ are all of them different, the curve is said to have a number of asymptotic directions equal to the degree of its equation. Such curves only will be considered in the present paper, the consideration of the far more complicated theory of those curves, the number of whose asymptotic directions is less than the degree of their equation, being entirely rejected. Assuming, then, that the factors of $H_{m}$ are all of them different, we may deduce from the equation $U=0$, by known methods, the series

$$
\begin{aligned}
& y=\alpha x+\alpha^{\prime}+\frac{\alpha^{\prime \prime}}{x}+\ldots \ldots \\
& y=\beta x+\beta^{\prime}+\frac{\beta^{\prime \prime}}{x}+\ldots \ldots \\
& \vdots \\
& y=\lambda x+\lambda^{\prime}+\frac{\lambda^{\prime \prime}}{x}+\ldots \ldots
\end{aligned}
$$

and these being obtained, we have, identically,

$$
U=\left(y-\alpha x-\alpha^{\prime}-\ldots\right)\left(y-\beta x-\beta^{\prime}-\ldots\right) \ldots\left(y-\lambda x-\lambda^{\prime}-\ldots\right)
$$


the negative powers of $x$ on the second side, in point of fact, destroying each other. Supposing in general that $f x$ containing positive and negative powers of $x, E f x$ denotes the function which is obtained by the rejection of the negative powers, we may write

$$
U=E\left(y-\alpha x \ldots-\frac{\alpha^{(m)}}{x^{m-1}}\right)\left(y-\beta x \ldots-\frac{\beta^{(m)}}{x^{m-1}}\right) \ldots\left(y-\lambda x \ldots-\frac{\lambda^{(m)}}{x^{m-1}}\right)
$$

the symbol $E$ being necessary in the present case, because, when the series are continued only to the power $x^{-m+1}$, the negative powers no longer destroy each other.

We may henceforward consider $U$ as originally given by the equation (3), the $m(m+1)$ quantities $\alpha, \alpha^{\prime} \ldots \alpha^{(m)}, \beta, \beta^{\prime} \ldots \beta^{(m)}, \ldots \lambda, \lambda^{\prime} \ldots \lambda^{(m)}$ satisfying the equations obtained from the supposition that it is possible to determine the following terms $\alpha^{(m+1)}, \beta^{(m+1)} \ldots \lambda^{(m+1)}, \ldots$ so that the terms containing negative powers of $x$, on the second side of equation (2), vanish. It is easily seen that $\alpha, \beta \ldots \lambda, \alpha^{\prime}, \beta^{\prime} \ldots \lambda^{\prime}$ are entirely arbitrary, $\alpha^{\prime \prime}, \beta^{\prime \prime} \ldots \lambda^{\prime \prime}$ satisfy a single equation involving only the preceding quantities, $\alpha^{\prime \prime \prime}, \beta^{\prime \prime \prime} \ldots \lambda^{\prime \prime \prime}$ two equations involving the quantities which precede them, and so on, until $\alpha^{(m)}, \beta^{(m)} \ldots \lambda^{(m)}$, which satisfy $(m-1)$ relations involving the preceding quantities. Thus the $m(m+1)$ quantities in question satisfy $\frac{1}{2} m(m-1)$ equations, or they may be considered as functions of $m(m+1)-\frac{1}{2} m(m-1)=\frac{1}{2} m(m+3)$ arbitrary constants. Hence the value of $U$, given by the equation (3), is the most general expression for a function of the $m^{\text {th }}$ order. It is to be remarked also that the quantities $\alpha^{(m+1)}, \beta^{(m+1)} \ldots \lambda^{(m+1)}, \ldots \ldots$ are all of them completely determinable as functions of $\alpha, \beta \ldots \lambda, \ldots \alpha^{(m)}, \beta^{(m)} \ldots \lambda^{(m)}$.

The advantage of the above mode of expressing the function $U$, is the facility obtained by means of it for the elimination of the variable $y$ from the equation $U=0$, and any other analogous one $V=0$. In fact, suppose $V$ expressed in the same manner as $U$, or by the equation

$$
V=E\left(y-A x \ldots-\frac{A^{(n)}}{x^{n-1}}\right)\left(y-B x \ldots-\frac{B^{(n)}}{x^{n-1}}\right) \ldots\left(y-K x \ldots-\frac{K^{(n)}}{x^{n-1}}\right)
$$

being the degree of the function $V$. It is almost unnecessary to remark, that $A, B \ldots K, \ldots A^{(n)}, B^{(n)} \ldots K^{(n)}$ are to be considered as functions of $\frac{1}{2} n(n+3)$ arbitrary constants, and that the subsequent $A^{(n+1)}, B^{(n+1)} \ldots K^{(n+1)} \ldots$ can be completely determined as functions of these. Determining the values of $y$ from the equation (3), viz. the values given by the equations (2); substituting these successively in the equation

$$
V=(y-A x-\ldots)(y-B x-\ldots) \ldots(y-K x-\ldots)=0
$$

analogous to (2), and taking the product of the quantities so obtained, also observing that this product must be independent of negative powers of $x$, the result of the elimination may be written down under the form

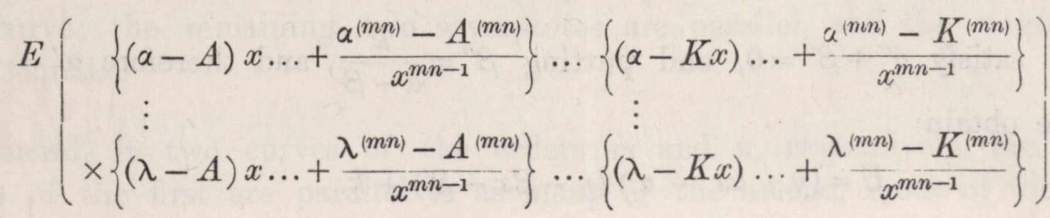


the series in \{\} being continued only to $x^{-m n+1}$, because the terms after this point produce in the whole product only terms involving negative powers of $x$. It is for the same reason that the series in ( ), in the equations (3) and (4), are only continued to the terms involving $x^{-m+1}, x^{-n+1}$ respectively.

The first side of the equation (6) is of the order $m n$, in $x$, as it ought to be. But it is easy to see, from the form of the expression, in what case the order of the first side reduces itself to a number less than $m n$. Thus, if $n$ be not greater than $m$, and the following equations be satisfied,

$$
\begin{array}{lll}
A=\alpha, & A^{(1)}=\alpha^{(1)} \ldots A^{(r-1)}=\alpha^{(r-1)}, & r \ngtr n \\
B=\beta, & B^{(1)}=\beta^{(1)} \ldots B^{(s-1)}=\beta^{(s-1)}, & s \ngtr r, \\
\vdots & \\
K=\kappa, & K^{(1)}=\kappa^{(1)} \ldots K^{(v-1)}=\kappa^{(v-1)}, & v \ngtr u,
\end{array}
$$

the degree of the equation (6) is evidently $m n-r-s \ldots-v$, or the curves $U=0, V=0$ intersect in this number only of points. If $m n-r-s \ldots-v=0$, the curves $U=0$ and $V=0$ do not intersect at all, and if $m n-r-s-v$ be negative, $=-\omega$ suppose, the equation (6) is satisfied identically; or the functions $U, V$ have a common factor, the number $\omega$ expressing the degree of this factor in $x, y$.

Supposing the function $V$ given arbitrarily, it may be required to determine $U$, so that the curves $U=0, V=0$ intersect in a number $m n-k$ points. This may in general be done, and done in a variety of ways, for any value of $k$ from unity to $\frac{1}{2} m(m+3)$. I shall not discuss the question generally at present, nor examine into the meaning of the quantity $m n-\frac{1}{2} m(m+3)\left\{=\frac{1}{2} m(2 n-m-3)\right\}$ becoming negative, but confine myself to the simple case of $U$ and $V$, both of them functions of the second order. It is required, then, to find the equations of all those curves of the second order which intersect a given curve of the second order in a number of points less than four.

Assume in general

$$
V=E\left(y-A x-A^{\prime}-\frac{A^{\prime \prime}}{x}\right)\left(y-B x-B^{\prime}-\frac{B^{\prime \prime}}{x}\right),
$$

then $A^{\prime \prime}, B^{\prime \prime}$ satisfy $A^{\prime \prime}+B^{\prime \prime}=0$, and putting $B^{\prime \prime}=\frac{K}{A-B}$, and therefore $A^{\prime \prime}=-\frac{K}{A-B}$ and reducing, we obtain

$$
V=\left(y-A x-A^{\prime}\right)\left(y-B x-B^{\prime}\right)+K .
$$

Similarly assume

$$
U=E\left(y-\alpha x-\alpha^{\prime}-\frac{\alpha^{\prime \prime}}{x}\right)\left(y-\beta x-\beta^{\prime}-\frac{\beta^{\prime \prime}}{x}\right) \text {, }
$$

then $\alpha^{\prime \prime}, \beta^{\prime \prime}$ satisfy $\alpha^{\prime \prime}+\beta^{\prime \prime}=0$, and putting $\beta^{\prime \prime}=\frac{k}{\alpha-\beta}$, and therefore $\alpha^{\prime \prime}=-\frac{k}{\alpha-\beta}$, and reducing, we obtain

$$
U=\left(y-\alpha x-\alpha^{\prime}\right)\left(y-\beta x-\beta^{\prime}\right)+k .
$$




\section{Suppose}

(1) $U=0, V=0$ intersect in three points, we must have $\alpha=A$, or the curve $U=0$ must have one of its asymptotes parallel to one of the asymptotes of $V=0$.

(2) The curves intersect in two points. We must have $\alpha=A, \alpha^{\prime}=A^{\prime}$, or else $\alpha=A, \beta=B$; i.e. $U=0$ must have one of its asymptotes coincident with one of the asymptotes of the curve $V=0$, or else it must have its two asymptotes parallel to those of $V=0$. The latter case is that of similar and similarly situated curves.

(3) Suppose the curves intersect in a single point only. Then either $\alpha=A$, $\alpha^{\prime}=A^{\prime}, \alpha^{\prime \prime}=A^{\prime \prime}$, which it is easy to see gives

$$
U=\left(y-A x-A^{\prime}\right)\left(y-\beta x-\beta^{\prime}\right)+K \frac{A-\beta}{A-B},
$$

or else $\alpha=A, \alpha^{\prime}=A^{\prime}, \beta=B$, which is the case of one of the asymptotes of the curve $U=0$, coinciding with one of those of the curve $V=0$, and the remaining asymptotes parallel. As for the first case, if $a, a_{1}$ are the transverse axes, $\theta, \theta_{1}$ the inclinations of the two asymptotes to each other, these four quantities are connected by the equation

$$
\frac{a^{2}}{a_{1}^{2}}=\frac{\tan \theta \cos ^{2} \frac{1}{2} \theta}{\tan \theta_{1} \cos ^{2} \frac{1}{2} \theta_{1}}
$$

and besides, one of the asymptotes of the first curve is coincident with one of the asymptotes of the second. This is a remarkable case; it may be as well to verify that $U=0, V=0$ do intersect in a single point only. Multiplying the first equation by $y-B x-B^{\prime}$, the second by $y-\beta x-\beta^{\prime}$, and subtracting, the result is

$$
(A-\beta)\left(y-B x-B^{\prime}\right)-(A-B)\left(y-\beta x-\beta^{\prime}\right)=0,
$$

reducible to

$$
y-A x=\frac{A\left(B^{\prime}-\beta^{\prime}\right)+B \beta^{\prime}-B^{\prime} \beta}{B-\beta} \text {, i.e. } y-A x-C=0 .
$$

Combining this with $V=0$, we have an equation of the form $y-B x-D=0$. And from this and $y-A x-C=0, x, y$ are determined by means of a simple equation.

(4) Lastly, when the curves do not intersect at all. Here $\alpha=A, \alpha^{\prime}=A^{\prime}, \beta=B$, $\beta^{\prime}=B^{\prime}$, or the asymptotes of $U=0$ coincide with those of $V=0$; i.e. the curves are similar, similarly situated, and concentric : or else $\alpha=A, \alpha^{\prime}=A^{\prime}, \alpha^{\prime \prime}=A^{\prime \prime}, \beta=B$; here

$$
U=\left(y-A x-A^{\prime}\right)\left(y-B x-\beta^{\prime}\right)+K,
$$

or the required curve has one of its asymptotes coincident with one of those of the proposed curve; the remaining two asymptotes are parallel, and the magnitudes of the curves are equal.

In general, if two curves of the orders $m$ and $n$, respectively, are such that $r$ asymptotes of the first are parallel to as many of the second, $s$ out of these asymptotes c. 
being coincident in the two curves, the number of points of intersection is $m n-r-s$; but the converse of this theorem is not true.

In a former paper, On the Intersection of Curves, [5], I investigated the number of arbitrary constants in the equation of a curve of a given order $\rho$ subjected to pass through the $m n$ points of intersection of two curves of the orders $m$ and $n$ respectively. The reasoning there employed is not applicable to the case where the two curves intersect in a number of points less than $m n$. In fact, it was assumed that, $W=0$ being the equation of the required curve, $W$ was of the form $u U+v V ; u, v$ being polynomials of the degrees $\rho-m, \rho-n$ respectively. This is, in point of fact, true in the case there considered, viz. that in which the two curves intersect in $m n$ points; but where the number of points of intersection is less than this, $u, v$ may be assumed polynomials of an order higher than $\rho-m, \rho-n$, and yet $u U+v V$ reduce itself to the order $\rho$. The preceding investigations enable us to resolve the question for every possible case.

Considering then the functions $U, V$ determined as before by the equations (3), (4), suppose, in the first place, we have a system of equations

$$
\alpha=A, \beta=B \ldots \ldots \theta=H \text { ( } t \text { equations) }
$$

Assume

$$
\begin{aligned}
& P=(y-\alpha x-\ldots)(y-\beta x-\ldots) \ldots(y-\theta x-\ldots), \\
& Q=(y-A x-\ldots)(y-B x-\ldots) \ldots(y-H x-\ldots) ; \\
& \Upsilon=(y-\iota x-\ldots) \ldots(y-\kappa x-\ldots), \\
& \Psi=(y-\mathrm{I} x-\ldots) \ldots(y-K x-\ldots) ;
\end{aligned}
$$

whence

$$
U=P \Upsilon, \quad V=Q \Psi .
$$

Suppose

$$
\Upsilon=E \Upsilon+\Delta \Upsilon, \quad \Psi=E \Psi+\Delta \Psi,
$$

$E \Psi \cdot U-E \Upsilon \cdot V=E \Psi \cdot P \Upsilon-E \Upsilon \cdot Q \Psi$

$$
\begin{aligned}
& =E \Psi \cdot P(E \Upsilon+\Delta \Upsilon)-E \Upsilon \cdot Q(E \Psi+\Delta \Psi), \\
& =E \Upsilon \cdot E \Psi \cdot(P-Q)+E \Psi \cdot P \cdot \Delta \Upsilon-E \Upsilon \cdot Q \cdot \Delta \Psi, \\
& =E\{E \Upsilon \cdot E \Psi \cdot(P-Q)+E \Psi \cdot P \Delta \Upsilon-E \Upsilon \cdot Q \cdot \Delta \Psi\}, \\
& =\Pi \text { suppose. }
\end{aligned}
$$

In this expression $E \Upsilon, E \Psi$ are of the degrees $m-t, n-t, \Delta \Upsilon, \Delta \Psi$ of the degree -1 , and $P, Q, P-Q$ of the degrees $t, t, t-1$ respectively. The terms of $\Pi$ are therefore of the degrees $m+n-t-1, m-1, n-1$ respectively, and the largest of these is in general $m+n-t-1$. Suppose, however, that $m+n-t-1$ is equal to $m-1$ (it cannot be inferior to it), then $t=n ; \Psi$ becomes equal to unity, or $\Delta \Psi$ vanishes. The remaining two terms of $\Pi$ are $E \Upsilon(P-Q), P \Delta \Upsilon$, which are of the degrees $m-1, n-1$ respectively. $\Pi$ is still of the degree $m-1$, supposing $m>n$. If $m=n$, the term $P \Delta \Upsilon$ vanishes. $\Pi$ is still of the degree $m-1$. Hence in every case the degree of $\Pi$ is $m+n-t-1$ : assuming always that $P-Q$ does not reduce itself to a degree lower than $t-1$, (which is always the case as long as the equations 
$\alpha^{\prime}=A^{\prime}, \beta^{\prime}=B^{\prime} \ldots \ldots \theta^{\prime}=H^{\prime}$ are not all of them satisfied simultaneously). It will be seen presently that we shall gain in symmetry by wording the theorem thus: the degree of $\Pi$ is equal to the greatest of the two quantities $m+n-t-1, m-1$.

Suppose next, in addition to the equations (8), we have

$$
\alpha^{\prime}=A^{\prime}, \quad \beta^{\prime}=B^{\prime} \ldots \ldots \zeta^{\prime}=F^{\prime}, \quad t^{\prime} \text { equations, } t^{\prime} \ngtr t \ldots\left(8^{\prime}\right) .
$$

Then, taking $\Upsilon^{\prime}, \Psi^{\prime}, P^{\prime}, Q^{\prime}$ the analogous quantities to $\Upsilon, \Psi, P, Q$, and putting

$$
E \Psi^{\prime} \cdot U-E \Upsilon^{\prime} \cdot V=\Pi^{\prime}
$$

we have, as before,

$$
\Pi^{\prime}=E\left\{E \Upsilon^{\prime} \cdot E \Psi^{\prime} \cdot\left(P^{\prime}-Q^{\prime}\right)+E \Psi^{\prime} \cdot P^{\prime} \Delta \Upsilon^{\prime}-E \Upsilon^{\prime} \cdot Q^{\prime} \Delta \Psi^{\prime}\right\} .
$$

The degree of $P^{\prime}-Q^{\prime}$ is $t^{\prime}-2$ (unless simultaneously $\alpha^{\prime \prime}=A^{\prime \prime}, \beta^{\prime \prime}=B^{\prime \prime} \ldots \ldots \zeta^{\prime \prime}=F^{\prime \prime}$, in which case the degree may be lower). The degrees, therefore, of the terms of $\Pi^{\prime}$ are $m+n-t^{\prime}-2, n-1, m-1$. Or we may say that the degree of $\Pi^{\prime}$ is equal to the greatest of the quantities $m+n-t^{\prime}-2, m-1$; though to establish this proposition in the case where $t^{\prime}=n-1$ would require some additional considerations.

Continuing in this manner until we come to the quantity $\Pi^{(k-1)}$, the degree of this quantity is the greatest of the two numbers $m+n-t^{(k-1)}-k, m-1$. And we may suppose that none of the equations $\alpha^{(k)}=A^{(k)} \ldots \ldots$ are satisfied, so that the series $\Pi, \Pi^{\prime} \ldots \ldots . \Pi^{(k-1)}$ is not to be continued beyond this point.

Considering now the equation of the curve passing through the $m n-t-t^{\prime} \ldots-t^{(k-1)}$ points of intersection of $U=0, V=0$, we may write

$$
W=u U+v V+p \Pi+p^{\prime} \Pi^{\prime} \ldots \ldots+p^{(k-1)} \Pi^{(k-1)}=0 .
$$

for the required equation; the dimensions of $u, v, p, p^{\prime} \ldots p^{(k-1)}$ being respectively

$p-m, \quad \rho-n ; \quad \rho-m-n+t+1$ or $\rho-m+1$

$$
\rho-m-n+t^{\prime}+2 \text { or } \rho-m+1 ; \ldots \ldots \rho-m-n+t^{(k-1)}+k \text { or } \rho-m+1 \text {, }
$$

the lowest of the two numbers being taken for the dimensions of $p, p^{\prime} \ldots p^{(k-1)}$. Also, any of these numbers become negative, the corresponding term is to be rejected. In saying that the degrees of $p, p^{\prime} \ldots \ldots . p^{(k-1)}$ have these actual values, it is supposed that the degrees of $\Pi, \Pi^{\prime} \ldots \ldots . \Pi^{k-1}$ actually ascend to the greatest of the values $\rho-m-n+t+1$, or $m-1 ; m+n-t^{\prime}-2$, or $m-1 ;-m+n-t^{(k-1)}+k$, or $m-1$.

The cases of exception to this are when several of the consecutive numbers $t, t^{\prime} \ldots t^{(k-1)}$ are equal. In this case the corresponding terms of the series $\Pi, \Pi^{\prime} \ldots \ldots . \Pi^{(k-1)}$, are also equal. Suppose for instance $t, t^{\prime}$ were equal, $\Pi, \Pi^{\prime}$ would also be equal. A term of $p$ of an order higher by unity than $\rho-m-n+t+1$, or $\rho-m+1$, which is the highest term admissible, produces in $p \Pi$ a term identical with one of the terms of $p^{\prime} \Pi$; so that nothing is gained in generality by admitting such terms into $p$. The equation (9), with the preceding values for the dimensions of $p, p^{\prime} \ldots \ldots p^{(k-1)}$, may be 
employed, therefore, even when several consecutive terms of the series $t, t^{\prime} \ldots \ldots t^{(k-1)}$ are equal. It will be convenient also to assume that $\rho-m$ is not negative, or at least for greater simplicity to examine this case in the first place.

$u, U$, and $v, V$, contain terms of the form $x^{a} y^{\beta} U, x^{\gamma} y^{\delta} V, \alpha+\beta \ngtr \rho-m, \gamma+\delta \ngtr \rho-n$; $p \Pi$ contains terms of this form, and in addition terms for which $\alpha+\beta=\rho+1-m$, $\gamma+\delta=\rho+1-n$. It is useless to repeat the former terms, so that we may assume for $p$, a homogeneous function of the order $\rho-m-n+t+1$, or $\rho-m+1$; in which case $p \Pi$ consists only of terms for which $\alpha+\beta=\rho+1-m, \gamma+\delta=\rho+1-n$. And the general expression of $p$ contains $\rho-m-n+t+2$, or $\rho-m+2$, arbitrary constants. Similarly $p^{\prime} \Pi^{\prime}$ contains terms of the form of those in $u U, v V, p \Pi$, and also terms for which $\alpha+\beta=\rho+2-m, \gamma+\delta=\rho+2-n$; the latter terms only need be considered, or $p^{\prime}$ may be assumed to be a homogeneous function of the order $\rho-m-n+t^{\prime}+2$, or $\rho-m+1$, containing therefore $\rho-m-n-t^{\prime}+3$, or $\rho-m+2$ arbitrary constants.

Similarly $p^{(k-1)}$ contains $\rho-m-n+t^{(k-1)}+k+1$ or $\rho-m+2$ arbitrary constants. Assume

$$
\nabla=\left(\begin{array}{l}
\rho-m-n+t+2 \\
\rho-m+2
\end{array}\right)+\left(\begin{array}{l}
\rho-m-n+t^{\prime}+3 \\
\rho-m+2
\end{array}\right) \ldots+\left(\begin{array}{l}
\rho-m-n+t^{(k-1)}+k+1 \\
\rho-m+2
\end{array}\right) \ldots
$$

where, in forming the value of $\nabla$ the least of the two quantities in ( ) is to be taken; this value also, if negative, being replaced by zero. The number of arbitrary constants in $p, p^{\prime} \ldots p^{(k-1)}$ is consequently equal to $\nabla$.

The numbers of arbitrary constants in $u, v$, are respectively

$$
\{1+2 \ldots \ldots+(\rho-m+1)\} \text { and }\{1+2 \ldots \ldots+(\rho-n+1)\}
$$

i.e.

$$
\frac{1}{2}(\rho-m+1)(\rho-m+2) \text {, and } \frac{1}{2}(\rho-n+1)(\rho-n+2) \text {; }
$$

thus the whole number of arbitrary constants in $W$, diminished by unity (since nothing is gained in generality, by leaving the coefficient (for instance of $y^{\rho}$ ) indeterminate, instead of supposing it equal to unity) becomes -

$$
\frac{1}{2}(\rho-m+1)(\rho-m+2)+\frac{1}{2}(\rho-n+1)(\rho-n+2)+\nabla-1,
$$

reducible to

$$
\frac{1}{2} \rho(\rho+3)+\frac{1}{2}(\rho-m-n+1)(\rho-m-n+2)-m n+\nabla .
$$

By the reasonings contained in the paper already referred to, if $\rho+k-m-n+1$ be positive, to find the number of really disposable constants in $W$, we must subtract from this number a number $\frac{1}{2}(\rho+k-m-n+1)(\rho+k-m-n+2)$. Hence, calling $\phi$ the number of disposable constants in $W$, we have

$$
\phi=\frac{1}{2} \rho(\rho+3)+\frac{1}{2}(\rho-m-n+1)(\rho-m-n+2)-m n+\nabla-\Lambda
$$

where

$$
\begin{aligned}
& \Lambda=0, \text { if } \rho+k-m-n+1 \text { be negative or zero. } \\
& \Lambda=\frac{1}{2}(\rho+k-m-n+1)(\rho+k-m-n+2),
\end{aligned}
$$

if $\rho+k-m-n+1$ be positive; and $\nabla$ is given by the equation (9). 
Also, if $\theta$ be the number of points through which the curve $W=0$ can be drawn, including the points of intersection of the curves $U=0, V=0$, then

$$
\begin{aligned}
& \theta=\phi+\left(m n-t-t^{\prime} \ldots \ldots-t^{(k-1)}\right) \text { or } \\
& \theta=\frac{1}{2} \rho(\rho+3)+\frac{1}{2}(\rho-m-n+1)(\rho-m-n+2)+\nabla-\Lambda-t-t^{\prime} \ldots-t^{(k-1)}
\end{aligned}
$$

Any particular cases may be deduced with the greatest facility from these general formuiæ. Thus, supposing the curves to intersect in the complete number of points $m n$, we have,

$$
\phi=\frac{1}{2} \rho(\rho+3)+\frac{1}{2}(1-8)(\rho-m-n+1)(\rho-m-n+2)-m n,
$$

8 being zero or unity according as $\rho<m+n-1$ or $\rho>m+n-1$. Reducing, we have, for $\rho \ngtr m+n-3$,

$$
\begin{aligned}
& \phi=\frac{1}{2} \rho(\rho+3)+\frac{1}{2}(\rho-m-n+1)(\rho-m-n+2)-m n, \\
& \theta=\frac{1}{2} \rho(\rho+3)+\frac{1}{2}(\rho-m-n+1)(\rho-m-n+2)
\end{aligned}
$$

and for $\rho>m+n-3$,

$$
\begin{aligned}
& \phi=\frac{1}{2} \rho(\rho+3)-m n, \\
& \theta=\frac{1}{2} \rho(\rho+3) .
\end{aligned}
$$

Suppose, in the next place, the curves have $t$ parallel pairs of asymptotes, none of these pairs being coincident. Then

$$
\begin{aligned}
& \rho \ngtr m+n-t-2, \\
& \quad \phi=\frac{1}{2} \rho(\rho+3)+\frac{1}{2}(\rho-m-n+1)(\rho-m-n+2)-m n, \\
& \theta=\frac{1}{2} \rho(\rho+3)+\frac{1}{2}(\rho-m-n+1)(\rho-m-n+2)-t ; \\
& \rho>m+n-t-2, \quad \rho \ngtr m+n-2, \\
& \quad \phi=\frac{1}{2} \rho(\rho+3)+\frac{1}{2}(\rho-m-n+2)(\rho-m-n+3)-m n+t, \\
& \theta=\frac{1}{2} \rho(\rho+3)+\frac{1}{2}(\rho-m-n+2)(\rho-m-n+3), \\
& \rho>m+n-2, \\
& \phi=\frac{1}{2} \rho(\rho+3)-m n+t, \\
& \theta=\frac{1}{2} \rho(\rho+3) .
\end{aligned}
$$

In these formulæ, if $t$ be equal to 2 or greater than 2 , the limiting conditions are more conveniently written

$$
\rho \ngtr m+n-t-2 ; \quad \rho \ngtr m+n-t-2>m+n-4 ; \quad \rho>m+n-4 .
$$

Similarly may the solution of the question be explicitly obtained when the curves have $t$ asymptotes parallel, and $t^{\prime}$ out of these coincident, but the number of separate formulæ will be greater.

In conclusion, I may add the following references to two memoirs on the present subject: the conclusions in one point of view are considerably less general even than those of my former paper, though much more so in another. Jacobi, Theoremata de punctis intersectionis duarum curvarum algebraicarum; Crelle's Journal, vol. xv. [1836, pp. 285-308]; Plücker, Théorèmes généraux concernant les equations à plusieurs variables, d'un degré quelconque entre un nombre quelconque d'inconnues. $\mathrm{D}^{\circ}$ vol. xvi. [1837, pp. $47-57]$. 


\section{Addition.}

As an exemplification of the preceding formulæ, and besides as a question interesting in itself, it may be proposed to determine the asymptotic curves of the $r^{\text {th }}$ order of a given curve having all its asymptotic directions distinct, $-r$ being any number less than the degree of the equation of the given curve.

Definition. A curve of the $r^{\text {th }}$ order, which intersects a given curve of the $m^{\text {th }}$ order in a number of points, $=m r-\frac{1}{2} r(r+3)$, is said to be an asymptotic curve of the $r^{\text {th }}$ order to the curve in question. Suppose, as before, $U=0$ being the equation to the given curve,

$$
U=E\left(y-\alpha x-\ldots-\frac{\alpha^{(m)}}{x^{m-1}}\right) \ldots\left(y-\lambda x \ldots-\frac{\lambda^{(m)}}{x^{m-1}}\right) ;
$$

and let $\theta, \phi \ldots \omega$ denote any combination of $r$ terms out of the series $\alpha \ldots \lambda$, and $\theta^{\prime}, \phi^{\prime} \ldots \omega^{\prime}$, $\& c . .$. the corresponding terms out of $\alpha^{\prime} \ldots \lambda^{\prime}$, \&c. Then, writing

$$
\begin{gathered}
V=E\left\{\left(y-\theta x \ldots-\frac{\theta^{(m)}}{x^{m-1}}\right)\left(y-\phi x \ldots-\frac{\phi^{(m-1)}}{x^{m-2}}-\frac{\Phi^{(m)}}{x^{m-1}}\right) \times \ldots \ldots\right. \\
\left.\left(y-\psi x \ldots-\frac{\dot{\psi}(m-2)}{x^{m-3}}-\frac{\Psi^{(m-1)}}{x^{m-2}}-\frac{\Psi^{(m)}}{x^{m-1}}\right) \ldots\left(y-\omega x-\Omega^{\prime} \ldots-\frac{\Omega^{(m)}}{x^{m-1}}\right)\right\},
\end{gathered}
$$

(where the quantities $\Phi^{(m)}, \Psi^{(m-1)}, \Psi^{(m)}, \Omega^{\prime} \ldots \Omega^{(m)}$ are entirely determinate, since, by what has preceded, $\theta^{\prime}, \phi^{\prime} \ldots \Omega^{\prime}$ satisfy a certain equation, $\theta^{\prime \prime}, \phi^{\prime \prime}, \ldots \Omega^{\prime \prime}$ two equations...... $\theta^{(m)}, \Phi^{(m)}, \ldots \Omega^{(m)}(m-1)$ equations), we have $V=0$ for the required equation of the asymptotic curve. It is obvious that the whole number of asymptotic curves of the order $r$, is $n(n-1) \ldots(n-r+1)$, viz. $1.2 \ldots r$ curves for each combination of $\frac{n(n-1) \ldots(n-r+1)}{1.2 \ldots r}$ asymptotes. Some particular instances of asymptotic curves will be found in a memoir by M. Plücker, Liouville's Journal, vol. I. [1836, pp. 229-252], Enumération des courbes du quatrième ordre, \&c. The general theory does not seem to be one to which much attention has been paid. 